\title{
DEFENSE INFORMATION SYSTEM NETWORK/NIPRNET MODELING AND ANALYSIS FOR UNCLASSIFIED ATM NETWORK
}

\author{
Dr. Savvas Hadjipanteli, Dr. Prabha Kumar, Sam Wang, \\ Defense Information Systems Agency, DISA/D84, C4I Systems Studies \& Analysis, \\ 701 S. Courthouse Road, Arlington, VA 22204 \\ E-mail: hadjipas@ncr.disa.mil, wangs@ncr.disa.mil
}

\begin{abstract}
Defense Information System Network (DISN) Model for ATM Services provides performance evaluation and prediction of the DISN ATM Services network. DISN/NIPRNET Modeling results for ATM Services show that the addition of the ATM network to DISN/NIPRNET CONUS (Continental US) network improves network performance. The system modeling and analysis was done mainly using Netmaker XA and MIIDAS tools with input traffic collected at individual nodes/routers in the network. Results and recommendations of this study are presented in the paper.
\end{abstract}

\section{INTRODUCTION}

DISN network is to provide the transmission infrastructure for integrated traffic of voice, video, and data for DoD services. The current tactical communication needs in terms of more bandwidth and more functionality is forcing the current tactical communications technology to search for improvements. These technology improvements using Commercial-OffThe-Shelf (COTS) and/or GovernmentOff-The-Shelf products is a long term
Department of Defense cost reduction strategy for modernizing the current tactical switches and communications devices. The goal is to have capabilities to deploy the most current commercial technology to the warfighter based on open/civil standards. This strategy allows the deployed tactical force to field current and emerging commercial technologies with minimal developmental cost.

A deployable tactical communications system using COTS technology/products was deployed as an ATM Infrastructure during JWID' 96 exercise [1]. This demonstration was about bandwidth economy and optimization. It provides a reliable example of maximizing the warfighters ability to manage and conserve scarce digital bandwidth entering and within the tactical area of operations $(\mathrm{AO})$. The increasing need for more bandwidth and cost effective services to support Command and Control requirements, Tele-medicine, and VTC (Video Conferencing) requirements makes the insertion of ATM technology in the existing DII (Defense Information Infrastructure) and DISN infrastructure very promising. The capability of the ATM technology to integrate different traffic types such as voice, video, and data onto one link allows effective 
bandwidth utilization, better performance, and cost savings [2-5]. In this work, DISN/NIPRNET modeling and analysis for the Unclassified-ATM (U-ATM) network was done using input traffic that was collected daily at individual nodes/routers interfaces. The Modular Information Infrastructure Design and Analysis System (MIIDAS) tool and the COTS tool, Netmaker XA, were the primary tools used for modeling and analysis in this work.

\section{SYSTEM MODEL AND TRAFFIC LOAD}

In the model, 75 sites and 121 intersite links were used to represent the NIPRNET-CONUS network topology. The model had also considered the additional intersite connectivity and additional bandwidth introduced by the U-ATM network. The U-ATM network was considered to be a fully meshed network of high bandwidth transmission links (DS3/OC-3 links) between 14 NIPRNET sites. The fully meshed network of 14 different nodes consists of 91 links. Figure 1 shows the geographic distribution of the DISN CONUS Bandwidth Managers (BWMs) and the NIPRNET routers that are directly connected to the DISN U-ATM network.

The knowledge of sufficient detailed description of the traffic that will be served by the network is necessary for a good network design, modeling, and analysis. An end-to-end traffic matrix was not available from measured traffic data for NIPRNET-CONUS. However, daily traffic statistics from and to each router interface were available. The average Kbps and packets per second (pps) sent to and from each router interface was available. The average offered traffic load to the system was about 18000 pps. Analysis of the data traffic had also showed that a significant percentage of this traffic is related to the Internet. Based on collected data samples, about $20 \%$ of the offered traffic was from the internet related routers and also about $20 \%$ of the offered traffic went to the internet related routers. The remaining traffic $(60 \%)$ originates and terminates at NIPRNET CONUS sites.

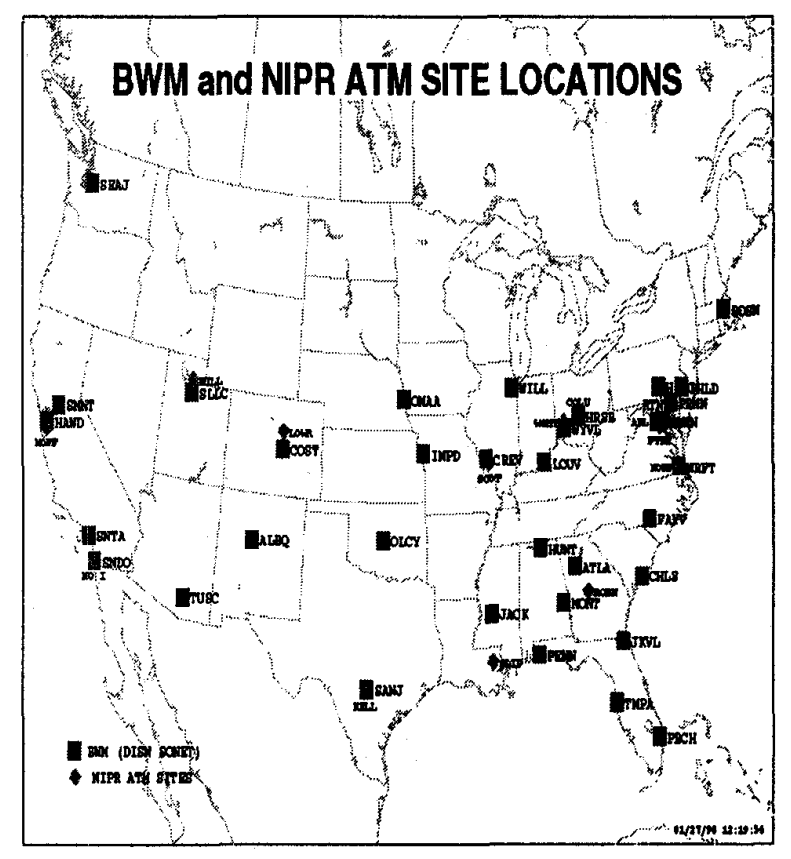

Figure 1. DISN CONUS BWMs and
NIPRNET ATM Sites

For input traffic considerations, each router site depending on service 
associated with, was categorized as A, $B, C$, or D. It was assumed that the majority of each site's traffic, non-JIS, about $75 \%$ was distributed to the other sites within the same category. To represent a busy network wide situation (war scenario), the average traffic load was doubled.

\section{RESULTS AND CONCLUSIONS}

\section{Baseline Network (ATM Services included):}

In this work, the Baseline Network (DISN/NIPRNET) consists of 75 backbone sites/nodes, 121 intersite connections, and the connectivity provided by the U-ATM network. The analysis results indicated that the network could tolerate about $15 \%$ of additional traffic increase before some links be fully saturated. For this network, The average number of Hops in the shortest path between sites is calculated to be 2.95 Hops and the Maximum number of Hops in the shortest path between sites to be 8 Hops. Considering traffic, the trafficweighted average number of Hops is calculated to be 1.96 Hops. (Importance was given here to paths that share more traffic). Summary Results are shown in Figure 2 and in Figure 3.

\section{Without ATM Services:}

Without the ATM services, without the U-ATM network, the results indicate an average number of Hops in the shortest path between sites to be 4.09 Hops and the Maximum number of Hops in the shortest path between sites to be 9.0 Hops. Considering traffic, the trafficweighted average number of Hops is calculated to be 3.36 Hops without ATM services. The summary results are shown in Figure 2 and in Figure 3.

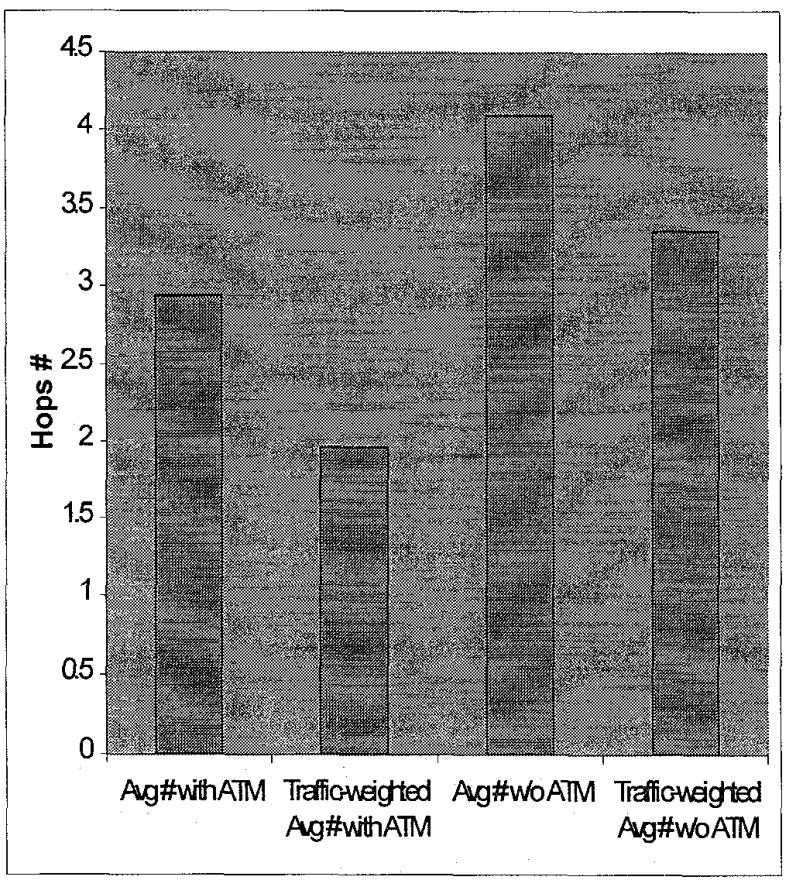

Figure 2. Average and Traffic-Weighted Average Number of Hops With ATM and Without ATM Network. 


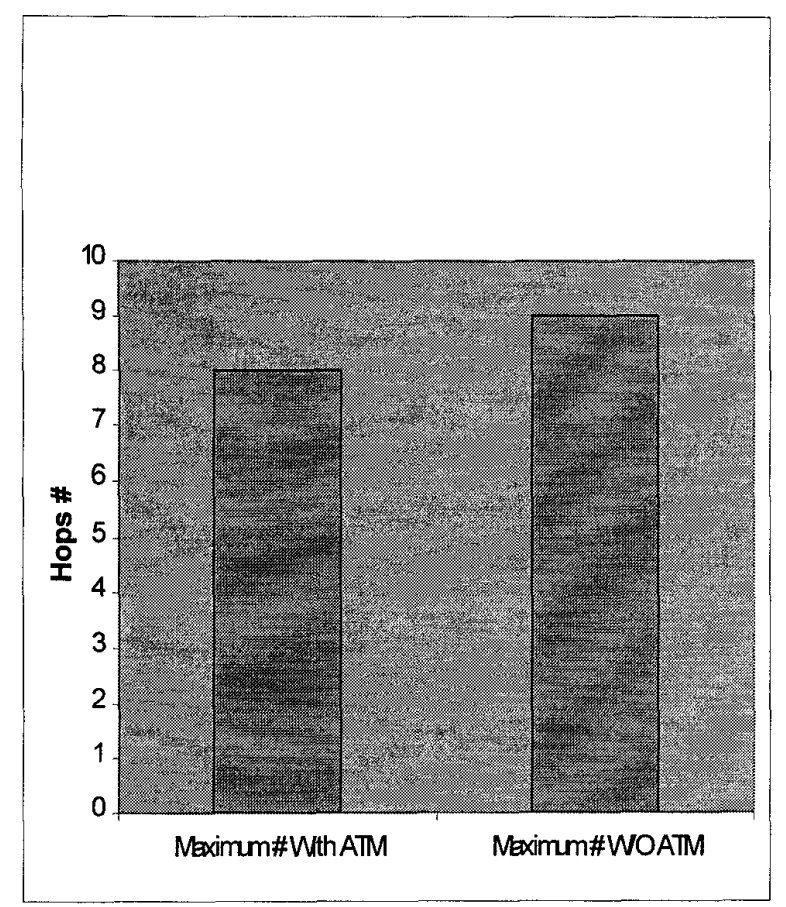

Figure 3. Maximum Number of Hops With ATM and Without ATM Network.

The above results and calculations indicate that the ATM services network provides performance improvement to the existing DISN/NIPRNET network. It provides about $25 \%$ decrease in the average number of Hops in the network when traffic is not considered and it provides about $40 \%$ decrease in the average number of Hops when traffic is considered. The addition of ATM services on the overall network improves also the Quality of Service and provides more bandwidth availability.

The Link-speed mismatch problem is still present in the system. For example, a $9.6 \mathrm{Kbps}$ link in the middle of paths mostly of $56 \mathrm{Kbps}$ links or a $56 \mathrm{Kbps}$ links in the middle of paths mostly of DS1 links. This problem is dominant for tactical communications where radio/wireless communications are widely used [6-8]. For additional performance improvement in the system, many Link-speed mismatches need to be eliminated.

NOTE: Opinions and recommendations that are mentioned in this paper reflect only the opinions of the authors and do not necessarily reflect the official policy and recommendations of DISA.

\section{REFERENCES:}

[1]. "Advanced Commercial-Off-TheShelf Technology For Joint Task Force DISN Deployed Multimedia Communications," Dr. Syed Shah, Terry Armstrong, Dr. Savvas Hadjipanteli, MILCOM 97, Monterey, CA.

[2]. "Integrated Information Management System For DISN ATM Services," Dr. Savvas Hadjipanteli, Dr. Seyd Shah, Larry Bowman, MILCOM 97, Monterey, CA.

[3]. "Effective Bandwidths For Multiclass Markov Fluids and Other ATM Sources," George Kesidis, Jean Walrand, Cheng-Shang Chang, IEEE Transactions on Networking, Vol. 1, No. 4, August 1993.

[4]. "Equivalent Capacity and Its Application to Bandwidth Allocation in High-Speed Networks," Roch Guerin, Hamid Ahmadi, Mahmoud Naghshineh, IEEE Journal on Selected Areas in Communications, Vol. 9, No. 7, 
September 1991.

[5]. "Taking Advantage of ATM Services and Tariffs: The Importance of Transport Layer Dynamic Rate Adaptation," Klaus-Jurgen Schulz, Marco Incollingo, and Hans Uhrig, IEEE Network, pp10-17, March/April 1997.

[6]. "Design and Performance Evaluation of RAC/EAC For Wireless ATM Environment," Ipkar Varshney, MILCOM 97, Monterey, CA.
[7]. "Multicasting Experiments in Tactical Operations," Earl Craighill and Jay Staba, MILCOM 97, Monterey, CA. [8]. "Multiband Multimode radio (MBMMR) Technology Applications in the Military, Civil, and Commercial Sectors," Dr. Eugene Newman and David M. Climek, MILCOM 97, Monterey, CA. 